\title{
Anisotropic Kondo pseudogap in $\mathrm{URu}_{2} \mathrm{Si}_{2}$
}

\author{
J. Buhot $\odot,{ }^{1,2,{ }^{*}}$ X. Montiel,,${ }^{3}$ Y. Gallais,,${ }^{2}$ M. Cazayous, ${ }^{2}$ A. Sacuto, ${ }^{2}$ G. Lapertot,${ }^{4}$ D. Aoki,${ }^{5,4}$ N. E. Hussey, ${ }^{1}$ C. Lacroix,${ }^{6}$ \\ C. Pépin, ${ }^{7}$ S. Burdin, ${ }^{8}$ and M.-A. Méasson ${ }^{6,2}$ \\ ${ }^{1}$ High Field Magnet Laboratory (HFML-EMFL), Institute for Molecules and Materials, Radboud University Nijmegen, Toernooiveld 7 , \\ 6525 ED Nijmegen, Netherlands \\ ${ }^{2}$ Laboratoire Matériaux et Phénomènes Quantiques, Université de Paris - CNRS, F-75013 Paris, France \\ ${ }^{3}$ Department of Physics, Royal Holloway, University of London, Egham, Surrey TW20 OEX, United Kingdom \\ ${ }^{4}$ University Grenoble Alpes, CEA, IRIG-Pheliqs, 38000 Grenoble, France \\ ${ }^{5}$ Institute for Materials Research, Tohoku University, Oarai, Ibaraki 311-1313, Japan \\ ${ }^{6}$ Université Grenoble Alpes, CNRS, Grenoble INP, Institut Néel, 38000 Grenoble, France \\ ${ }^{7}$ Institut de Physique Théorique, CEA-Saclay, 91191 Gif-sur-Yvette, France \\ ${ }^{8}$ Université Bordeaux, CNRS, LOMA, UMR 5798, 33400 Talence, France
}

(Received 3 May 2018; revised manuscript received 18 April 2020; accepted 12 May 2020; published 1 June 2020)

\begin{abstract}
A polarized electronic Raman scattering study reveals the emergence of symmetry dependence in the electronic Raman response of single-crystalline $\mathrm{URu}_{2} \mathrm{Si}_{2}$ below the Kondo crossover scale $T_{K} \sim 100 \mathrm{~K}$. In particular, the development of a coherent Kondo pseudogap predominantly in the $E_{g}$ channel highlights strong anisotropy in the Kondo physics in $\mathrm{URu}_{2} \mathrm{Si}_{2}$ that was previously neglected in theoretical models of this system and more generally has been sparsely treated for Kondo systems. A calculation of the Raman vertices demonstrates that the strongest Raman vertex does, indeed, develop within the $E_{g}$ channel for interband transitions and reaches a maximum along the diagonals of the Brillouin zone, implying a $d$-wave-like geometry for the Kondo pseudogap. This generally overlooked property may have important consequences on the formation of the subsequent phases of this system, namely, the hidden order and the superconductivity.
\end{abstract}

DOI: 10.1103/PhysRevB.101.245103

\section{INTRODUCTION}

The Kondo effect, in which an antiferromagnetic coupling between conduction electrons and local quantum impurities leads to the formation of a Kondo singlet, is well understood for single Kondo impurity systems and is characterized by the appearance of a Kondo resonance in the electronic density of states below a crossover temperature $T_{K}$ [1]. By contrast, Kondo lattice systems, which are realized when the Kondo rare-earth ions form a regular lattice, appear much more complex [2] and exhibit surprising phenomena [3,4]. Usually, the microscopic state of heavy fermions is described by Anderson or Kondo lattice models [2], and most of the time the Kondo singlet state is considered to be fully isotropic ( $s$-wave symmetry with zero angular momentum). In certain circumstances, however, a momentum-dependent Anderson hybridization, for which the $f$ site hybridizes with its nearestneighbor conduction sites $[5,6]$, or a nonlocal Kondo coupling [7] can give rise to the formation of heavy quasiparticles with a strong $k$ dependence; in such a case the singlet state may have a higher angular momentum rank $(p, d, \ldots)$ or may be of extended $s$ type [6]. As a consequence, the Kondo gap can instead be a pseudogap (partial gapping of the Fermi surface) that develops in the coherent Kondo regime [8-11]; this pseu-

*Present address: HH Wills Laboratory, University of Bristol, Bristol BS8 1TL, United Kingdom; jonathan.buhot@bristol.ac.uk dogap is anisotropic in $k$ space and might exhibit nodal and antinodal regions. The possible symmetries for the effective Kondo hybridization are defined in analogy with the gap symmetries in strongly correlated superconductors (cuprates, heavy fermions, Fe pnictides). In Kondo systems, reports of such momentum-dependent hybridization are scarce [12] and remain indirect.

A nonlocal Kondo hybridization may be particularly relevant for the complex Kondo system $\mathrm{URu}_{2} \mathrm{Si}_{2}$ where an enigmatic "hidden"-order (HO) phase appears inside the Kondo coherent regime below $T_{H O}=17.5 \mathrm{~K}$ and an unconventional superconducting (SC) phase develops below $T_{c}=$ $1.5 \mathrm{~K}[13,14]$. Many spectroscopic experiments have reported a Kondo pseudogap opening at a temperature scale $T_{K}=$ $50-100 \mathrm{~K}$, well above $T_{H O}$, suggesting that Kondo hybridization is not the cause of the $\mathrm{HO}$ transition [15-20]. On the other hand, scanning tunneling microscopy (STM) has revealed an asymmetric pseudogap opening at $T_{H O}$ within the KondoFano lattice structure that appears below $T_{K}$ [21,22]. The HO gap is found to be correlated on the atomic scale with the electronic signatures of the Kondo lattice state, suggesting that the two phenomena involve the same electronic states. Angleresolved photoemission spectroscopy (ARPES), meanwhile, has shown that a Kondo pseudogap opens at the $X$ point of the Brillouin zone, while the HO gap affects electronic states at the $\Gamma$ and $Z$ points [23]. Nevertheless, the electronic structure in the $\mathrm{HO}$ state at the $Z$ point (M-shaped dispersion) results from the hybridization of a light electron band 
with already hybridized bands characterizing the paramagnetic state. Moreover, high magnetic field measurements have demonstrated that the destabilization of the HO state (around $37 \mathrm{~T})$ coincides with the "collapse" of the Kondo crossover $\left(T_{\rho, \max }, H_{\rho, \max } \rightarrow 0\right)$ [24].

Significant theoretical works have been performed to identify the correct order parameter for the HO state [14]. Some theories consider the Kondo hybridization an important ingredient in the realization of the HO [25-29], with others even proposing the hybridization as the order parameter of the $\mathrm{HO}$ state itself [30,31]. Moreover, under a pressure of $\sim 0.5 \mathrm{GPa}$, the SC and HO states collapse together [13], promoting the $\mathrm{HO}$ as a precursor of SC pairing possibly of a chiral $d$-wave type [32-34]. The relationship between the various phases of $\mathrm{URu}_{2} \mathrm{Si}_{2}$ is still an open question. Here, we unveil a generally overlooked property of the Kondo physics of $\mathrm{URu}_{2} \mathrm{Si}_{2}$, i.e., its anisotropic character, which is not only relevant to this question for this complex system but also sparsely discussed in the broad field of Kondo physics.

Here, we present Raman polarized spectroscopy measurements in both the Kondo phase and the $\mathrm{HO}$ phase of $\mathrm{URu}_{2} \mathrm{Si}_{2}$. We observe a Kondo pseudogap opening only in the $E_{g}$ symmetry below $T_{K}$. These results, together with our accompanying Raman calculations, highlight a strong anisotropy in the Kondo physics in $k$ space and reveal a $d$-wave-like geometry for the Kondo pseudogap.

\section{EXPERIMENTAL DETAILS}

Polarized Raman experiments were carried out using a solid-state laser emitting at $532 \mathrm{~nm}$ and a $\mathrm{K}^{+}$laser emitting at $647 \mathrm{~nm}$. The scattered light was analyzed by a Jobin Yvon T64000 simple grating spectrometer equipped with an ultrasteep long-pass edge filter to reject the Rayleigh scattering. Single crystals of $\mathrm{URu}_{2} \mathrm{Si}_{2}$ were grown by the Czochralski method using a tetra-arc furnace [35]. All the $D_{4 h}$ point group (space group number 139) symmetries [36] have been measured. To probe the $E_{g}$ symmetry, we have used samples polished along the $a c$ plane. For completeness, $A_{1 g}, A_{2 g}, B_{1 g}$, and $B_{2 g}$ symmetries have been explored in samples freshly cleaved along the $a b$ plane. Temperature-dependent studies were carried out in a closed-cycle ${ }^{4} \mathrm{He}$ cryostat with the sample in vacuum. All reported temperatures are corrected for laser heating following Refs. [37-40].

\section{RESULTS AND DISCUSSION}

Figure 1 presents the Raman spectra of $\mathrm{URu}_{2} \mathrm{Si}_{2}$ in the $E_{g}$ and $A_{1 g}+B_{2 g}$ symmetries. For the $E_{g}$ symmetry [Fig. 1(a)], the sharp peaks at 210 and $390 \mathrm{~cm}^{-1}$ are the two $E_{g}$ phonon modes [41], while the small peak at $430 \mathrm{~cm}^{-1}$ is a leakage of the $A_{1 g}$ phonon mode. The bump at $\sim 800 \mathrm{~cm}^{-1}$ has been associated with a crystal electric field excitation [42]. In the $\left[A_{1 g}+B_{2 g}\right]$ symmetry [Fig. 1(b)], the intense $A_{1 g}$ phonon mode is observed at $\sim 430 \mathrm{~cm}^{-1}$. Smaller features seen at 350 , 760 , and $832 \mathrm{~cm}^{-1}$ are related to double-phonon processes [42]. Finally, tiny leakages of $B_{1 g}$ and $E_{g}$ phonon modes are visible at 165,210 , and $390 \mathrm{~cm}^{-1}$.

Remarkable effects are observed in the electronic continuum below $\sim 700 \mathrm{~cm}^{-1}(\sim 90 \mathrm{meV})$ in both the $E_{g}$ and $A_{1 g}$
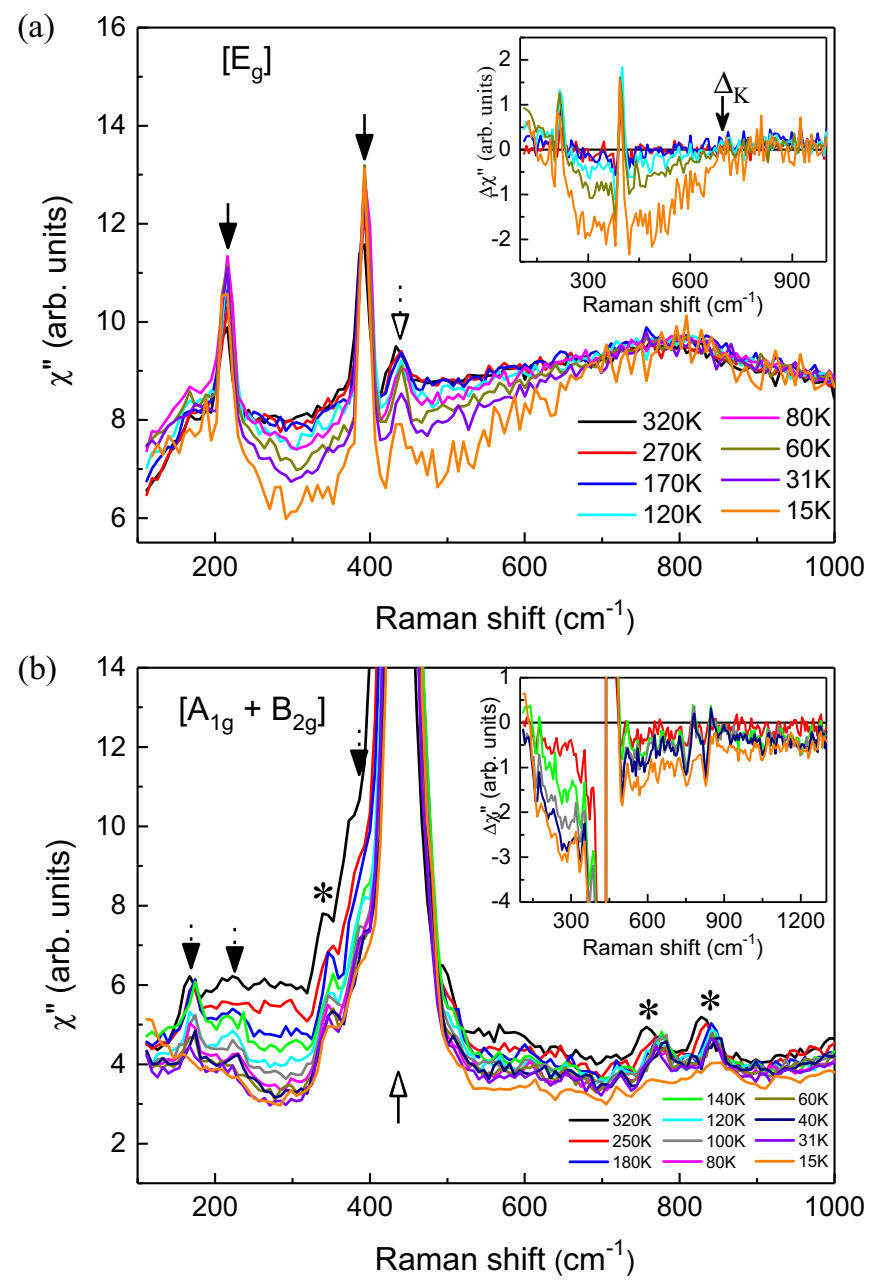

FIG. 1. Raman spectra of $\mathrm{URu}_{2} \mathrm{Si}_{2}$ in the (a) $E_{g}$ and (b) $A_{1 g}+$ $B_{2 g}$ symmetries. The susceptibilities $\chi^{\prime \prime}$ are normalized to the laser power. Solid and open arrows indicate, respectively, the $E_{g}$ and $A_{1 g}$ phonon modes. Dotted arrows indicate leakages of phonon modes. The symbol $*$ denotes double-phonon processes [42]. Insets show the subtracted Raman responses $\Delta \chi^{\prime \prime}=\chi^{\prime \prime}(T)-\chi^{\prime \prime}\left(T_{320 K}\right)$. The laser line wavelength used is $532 \mathrm{~nm} . \Delta_{K}$ corresponds to the energy below which the Kondo pseudogap opens.

symmetries, i.e., a depletion in a large energy range upon cooling. As shown in Figs. 2(a) and 2(b), such a depletion is absent in the $B_{1 g}, B_{2 g}$, and $A_{2 g}$ channels. In Fig. 2(c), we illustrate this loss of spectral weight by integrating $\Delta \chi^{\prime \prime}(T)=$ $\chi^{\prime \prime}(T)-\chi^{\prime \prime}(320 \mathrm{~K})$ from the lowest energy $\left(100 \mathrm{~cm}^{-1}\right)$ to $1500 \mathrm{~cm}^{-1}$ for all symmetries. An example of the raw data is presented in the inset of Fig. 2(c). The large error bars in the $\left[B_{1 g}+B_{2 g}\right]$ channel prohibit us from drawing any definitive conclusions for the $B_{2 g}$ symmetry. Nevertheless, its spectral weight loss is found to be weaker than that for the $E_{g}$ response. This loss of spectral weight happens from $320 \mathrm{~K}$ in both $E_{g}$ and $A_{1 g}$ symmetries. Below $100 \mathrm{~K}$, however, the depletion in the $E_{g}$ symmetry begins to accelerate, while for $A_{1 g}$, it decreases at a constant rate. This temperature scale of $100 \mathrm{~K}$ matches with $T_{K}[21,22,43,44]$.

Moreover, at the Kondo crossover a direct pseudogap, i.e., a partial depletion in the electronic continuum not associated 

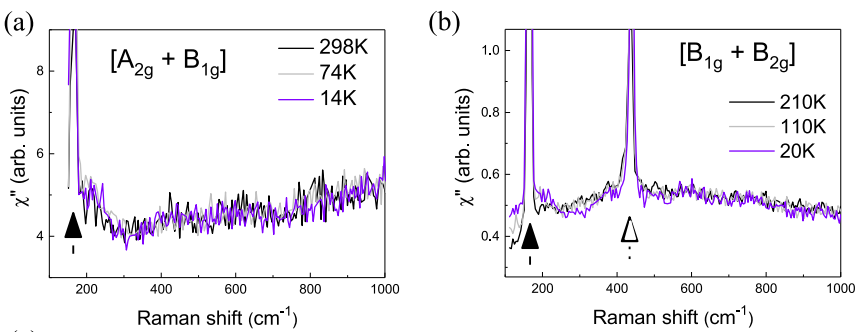

(c)

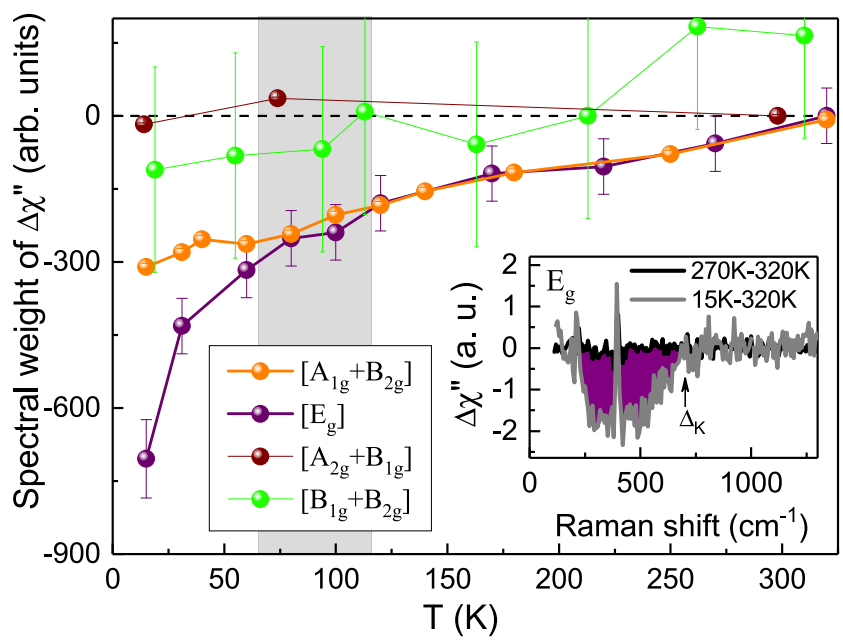

FIG. 2. Raman spectra of $\mathrm{URu}_{2} \mathrm{Si}_{2}$ in the (a) $\left[A_{2 g}+B_{1 g}\right]$ and (b) $\left[B_{1 g}+B_{2 g}\right]$ symmetries. The laser line wavelengths are 647 and $532 \mathrm{~nm}$, respectively. Open arrows with a dotted line and solid arrows with a dashed line indicate, respectively, the $A_{1 g}$ (leakage) and $B_{1 g}$ phonon modes. (c) Temperature dependence of normalized spectral weights of subtracted Raman responses $\Delta \chi^{\prime \prime}=\chi^{\prime \prime}(T)-$ $\chi^{\prime \prime}\left(T_{\text {ambiante }}\right)$ in $\left[A_{2 g}+B_{1 g}\right],\left[B_{1 g}+B_{2 g}\right],\left[A_{1 g}+B_{2 g}\right]$, and $\left[E_{g}\right]$ symmetries. Error bars for $\left[A_{1 g}+B_{2 g}\right]$ symmetry are smaller than the data dots. The inset shows an example of how the spectral weight was extracted in the $E_{g}$ symmetry, in this case at $T=15 \mathrm{~K}$. A Kondo pseudogap opens below $\sim 100 \mathrm{~K}$ only in the $E_{g}$ symmetry.

with any symmetry breaking, or even a full direct gap for a Kondo insulator [45] is expected to naturally emerge due to the hybridization of the localized and itinerant bands. This has been generally observed in Kondo systems by optical conductivity $[46,47]$. Such depletion in the electronic background should also naturally be measured by Raman spectroscopy even if, up to now, observations have been limited to Kondo insulator systems [48]. In the $\mathrm{URu}_{2} \mathrm{Si}_{2}$ compound, we ascribe the Raman depletion in the $E_{g}$ symmetry to the Kondo pseudogap because it is also present in optical conductivity measurements below the same energy scale [15-20] and with a similar drop below $T_{K}$ [49]. Thus, our observation constitutes the first measurement of a Kondo pseudogap in a metallic system by Raman spectroscopy. Most interesting is the symmetry dependence of the Raman pseudogap since it implies a strong anisotropy in the Kondo physics in $\mathrm{URu}_{2} \mathrm{Si}_{2}$. Optical conductivity also shows anisotropic behavior between the $a b$-plane and $c$-axis responses [20,49]. The $a b$-plane optical conductivity is strongly influenced by the formation of coherent Kondo singlets, reminiscent of what happens in the $E_{g}$ Raman response, while the $c$-axis optical conductivity decreases continuously below $40 \mathrm{meV}$ with no pseudogap sig- natures through the Kondo crossover, mimicking the Raman response seen in the $A_{1 g}$ channel.

While a single energy scale $\Delta_{K}=700 \mathrm{~cm}^{-1}$ for the pseudogap is identified in both $E_{g}$ and $A_{1 g}$ symmetries [see inset in Fig. 2(c)], the temperature dependence of the Raman susceptibilities in these symmetries reveals two distinct temperature scales: one above $320 \mathrm{~K}\left(T_{K}^{\text {high }}\right)$ and a second at $T_{K}$. High-temperature Kondo $\left(T_{K}^{\text {high }}>T_{K}\right)$ behavior was observed previously in transport measurements at $\sim 370 \mathrm{~K}$ and was associated with the formation of incoherent Kondo singlets [50]. $T_{K}^{\text {high }}$ may also result from a Kondo coherence effect similar to what happens at $T_{K}$ but with a larger effective degeneracy of the $f$ orbitals due to thermal activation of the crystal electric field states. This would then give a second value of coherence $T_{K}^{\text {high }}>T_{K}$. Indeed, it has been shown that $T_{K}$ for a multifold-degenerate (for instance, with a degeneracy of 4) Kondo model is significantly higher than $T_{K}$ computed for the twofold-degenerate equivalent model $[2,8]$. Thus, a similar Kondo description could interpolate smoothly, from multifold-degenerate impurities below $T_{K}^{\text {high }}$ to twofold degenerate impurities below $T_{K}$. In such a description, at high temperature, where more orbitals are involved, the Kondo mechanism would give rise to more isotropic effects than at low temperature where the orbital selectivity is strengthened. Consequently, the Kondo physics may be observable in several Raman-active symmetries below $T_{K}^{\text {high }}$ (here in both $E_{g}$ and $A_{1 g}$ symmetries) and strengthen only in one specific symmetry ( $E_{g}$ in the present case) below $T_{K}$. Recently, such orbital selectivity for the Kondo effect was, indeed, observed with different experimental techniques in $\mathrm{USb}_{2}[51,52]$.

The energy scale for the spectral weight depletion in the $E_{g}$ and $A_{1 g}$ symmetries is quite consistent with the previous observations of a Kondo pseudogap in $\mathrm{URu}_{2} \mathrm{Si}_{2}$ at $\sim 500 \mathrm{~cm}^{-1}$ by optical conductivity measurements [15-18,49] or at $\sim 250$ $\mathrm{cm}^{-1}$ by STM measurements [21,22]. ARPES [23] and quasiparticle scattering spectroscopy [19] reported a lower hybridization pseudogap at $11 \mathrm{meV}\left(\sim 90 \mathrm{~cm}^{-1}\right)$. In Kondo models [8-11,53], the electronic dispersion is characterized by a direct pseudogap (with zero transferred momentum) of the order of an effective hybridization $\Delta$ and an indirect pseudogap $\Delta^{2} / W \sim k_{B} T_{K}$, where $W$ is the electronic bandwidth. Raman spectroscopy probes the direct Kondo pseudogap, while, for example, ARPES measures the indirect Kondo pseudogap. In the present case, $\Delta \sim \Delta_{K}=700 \mathrm{~cm}^{-1}$, and if we assume $W=1 \mathrm{eV}$, we extract an indirect pseudogap of $7.5 \mathrm{meV}\left(\sim 60 \mathrm{~cm}^{-1}\right)$, in agreement with the ARPES measurements. From these phenomenological considerations, the relevant temperature scale is found to be of the order of $T_{K}$. Interestingly, this description also accounts for the very large ratio between the Kondo direct pseudogap energy and the Kondo crossover temperature $\frac{\Delta_{K}}{k_{B} \cdot T_{K}}$. This ratio is of the order of 10 in $\mathrm{URu}_{2} \mathrm{Si}_{2}$, as found in Kondo insulators $[54,55]$.

In general and in contrast to optical conductivity measurements, electronic Raman scattering is not expected to follow spectral weight sum rules. However, some models show that interaction-dependent sum rules are possible [56]. For instance, in Kondo insulators such as FeSi and $\mathrm{SmB}_{6}$, Raman electronic spectral weight that is suppressed at low 
frequencies by the charge gap is primarily recovered within an energy range equal to $6 \Delta$. This has been discussed theoretically in the framework of the Hubbard model [55] and extended to metallic systems for which a clear redistribution of spectral weight to a Fermi-liquid peak at low energy or, in some cases, to a high-energy charge-transfer peak is expected [57]. However, according to our present and previous [58] results, $\mathrm{URu}_{2} \mathrm{Si}_{2}$ does not show any Raman spectral weight redistribution either above or below the depletion energy scale, which seems to indicate that the $\mathrm{URu}_{2} \mathrm{Si}_{2}$ Raman response does not follow any particular sum rule. Thus, our results call for further theoretical investigations in the context of Kondo metallic systems.

The $E_{g}$ symmetry dependence of the Raman electronic continuum presented above implies $k$-space anisotropy in the quasiparticle excitations. Via the Raman vertex [59], polarized Raman scattering has the ability to provide information on the different regions of the Brillouin zone probed for each selected symmetry. In order to elucidate the $k$-space dependence of the Kondo physics, we have therefore carried out Raman vertex calculations within the effective-mass approximation $[59,60]$.

The intraband and interband Raman vertices have been calculated using a tight-binding electronic band structure developed on the $5 f^{2}$ uranium electron's configuration for $\mathrm{URu}_{2} \mathrm{Si}_{2}$ [61,62]. This model is based on an effective one-particle picture with the $J=5 / 2$ states. The crystal electric field effect splits this sextet into three Kramers doublets $\Gamma_{7}^{(1)} \oplus \Gamma_{7}^{(2)} \oplus \Gamma_{6}$. For simplicity, the $\Gamma_{6}$ doublet is neglected. The main contribution to the Fermi surface comes from the two $\Gamma_{7}$ doublets that are described in the tight-binding approximation. Here, the Kondo lattice effect is considered a mixing between the two $\Gamma_{7}$ doublets whose contribution to the Hamiltonian depends on a hopping term $t_{12}$ between uranium sites at the zone center and at the corners. The body-centered tetragonal (bct) Brillouin zone of the paramagnetic state is shown in Fig. 3(a). The Fermi surface obtained [Fig. 3(b)] from the electronic dispersion [Fig. 3(c)] is consistent with earlier $a b$ initio calculations [63]. Only the small electrons pockets at $\Gamma$ and $X$ [64] are not reproduced by this simple model [62]. The electronic dispersion does not depend strongly on the hopping term $t_{12}$ except around the $N$ point, where the energy between the two $\Gamma_{7}$ bands increases when $t_{12}$ deviates from zero.

From our calculations, large values of the Raman vertices were found in the $E_{g}$ and $A_{1 g}$ symmetries, while in the $B_{1 g}$ and $B_{2 g}$ symmetries, the vertices remain very weak. The strongest value of the Raman vertex is, in fact, found in the $E_{g}$ symmetry for interband transitions. The $E_{g}$ vertex for intraband transitions, on the other hand, is weak. According to this result, the $E_{g}$ Raman response mainly comes from vertical interband transitions along the diagonals of the Brillouin zone. Indeed, as emphasized in Fig. 3(b), the interband $E_{g}$ Raman vertex is strong only at the $X, Y$, and $Y_{1}$ points [as indicated by solid arrows in Fig. 3(c)]. Consequently, the Kondo pseudogap is linked to the electronic states at these $k$ points, reflecting the $d$-wave like geometry of the Kondo pseudogap.

The $A_{1 g}$ interband vertex is particularly strong at the $N$ point where the interband vertical electronic transition has typically the same energy scale. The Raman electronic depletion change is smooth at $T_{K}$ in this symmetry, thus confirming (a)

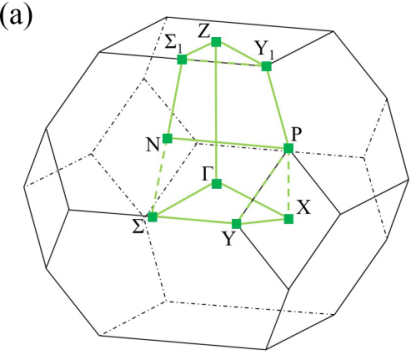

(b) $\mathrm{E}_{\mathrm{g}}$ interband vertex

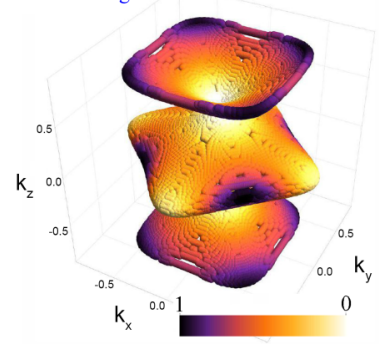

(c)

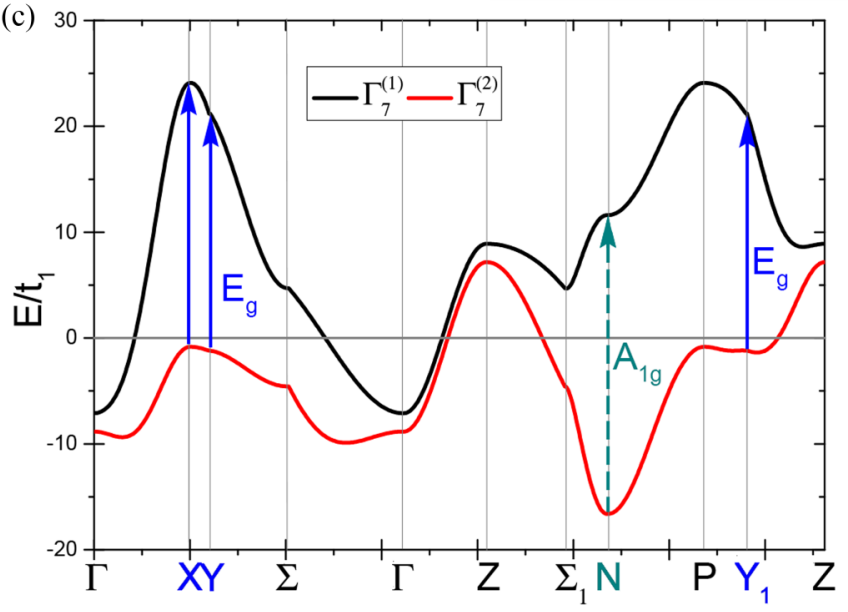

FIG. 3. (a) Body-centered tetragonal Brillouin zone of $\mathrm{URu}_{2} \mathrm{Si}_{2}$. (b) Relative magnitude of the $E_{g}$ Raman vertex at the Fermi surface for interband transitions calculated within the effective-mass approximation. (c) Calculated electronic dispersion of the $\Gamma_{7}^{(1)}$ and $\Gamma_{7}^{(2)}$ bands including hybridization. The solid (dashed) arrows at the $X, Y$, and $Y_{1}(N)$ locations indicate the Brillouin zone points where the $E_{g}\left(A_{1 g}\right)$ interband vertex reaches a maximum value (see text).

that the Kondo pseudogap does not affect the $N$ point of the Brillouin zone, another indication of strong anisotropy in the Kondo physics in $\mathrm{URu}_{2} \mathrm{Si}_{2}$.

We now turn to discuss the relationship between the $\mathrm{HO}$ state and the Kondo physics in light of our Raman results. The main observations are twofold: the Kondo pseudogap in the $E_{g}$ channel seems to be reinforced below $T_{H O}$ (it is most significant between 30 and $15 \mathrm{~K}$ ), suggesting that there is no sign of suppression of the Kondo effect upon entering the HO state [27]. Moreover, our experimental results reveal no pseudogap opening in the $A_{2 g}$ symmetry despite the fact that this peculiar symmetry has been shown to exhibit all the Raman signatures of the $\mathrm{HO}$ state, including a sharp excitation (at $1.7 \mathrm{meV}$ ) and a gap (at $6.8 \mathrm{meV}$ ) [58,65]. Raman experiments $[58,66]$ also showed that the width of the $A_{2 g}$ quasielastic continuum in the paramagnetic state is affected by the Kondo crossover, suggesting that the $A_{2 g}$ Raman channel is sensitive to the hybridization process. A definitive conclusion could be reached by going beyond the effective-mass approximation so that the $A_{2 g}$ Raman vertex is not expected to be zero. This would provide the $k$-dependent variation in the $A_{2 g}$ Raman response, notably the $\mathrm{HO} A_{2 g}$ gap below $6.8 \mathrm{meV}$, which may also have a $d$-wave geometry and, as a result, may be favorable to the chiral $d$-wave $k_{z}\left(k_{x}+i k_{y}\right)\left(E_{g}\right)$ pairing proposed for the SC state [32-34]. 
Interestingly, in a two-channel Kondo model such as the hastatic order model [31], the normal Kondo crossover at $T_{K}$ may be converted into a phase transition at $T_{H O}$. Multiple components for the hybridization gap with different symmetries are expected: one that could open at $T_{K}$ (as a crossover without symmetry breaking) and one that should manifest at $T_{H O}$ as an order parameter for the HO [67]. A model inspired by a similar scenario might reconcile the $E_{g}$ symmetry of the Kondo pseudogap with the $A_{2 g}$ symmetry of the HO gap.

\section{CONCLUSION}

In summary, we report the observation of an anisotropic Kondo pseudogap in a metallic Kondo lattice system by Raman spectroscopy. We observe a pseudogap opening within the $E_{g}$ symmetry in $\mathrm{URu}_{2} \mathrm{Si}_{2}$ that appears to be linked to the Kondo crossover below $100 \mathrm{~K}$. From Raman vertex calculations, the interband Raman vertex in the $E_{g}$ symmetry is strongest at the $X, Y$, and $Y_{1}$ points of the Brillouin zone, suggesting that the Kondo pseudogap has a $d$-wavelike symmetry. This anisotropy in the Kondo physics may play a significant role in the realization of the hidden order in $\mathrm{URu}_{2} \mathrm{Si}_{2}$ and henceforth should be considered a key element in further theoretical developments. Generally, it may be a generic property of some Kondo lattice systems with an important impact on their entire phase diagrams. Raman spectroscopy, as a $k$-dependent probe, is proven to be well suited for such investigations.

\section{ACKNOWLEDGMENTS}

This work was supported by the French Agence Nationale de la Recherche (ANR "PRINCESS" Grant No. ANR-11BS04-0002, ANR-DFG "Fermi-NEst" Grant No. ANR-16CE92-0018, ANR "SEO-HiggS2" Grant No. ANR-16-CE300014). We thank J. G. Rau, H.-Y. Kee, P. Thalmeier, L. H. Tjeng, A. Severing, S. Wirth, and R. Lobo for very fruitful discussions.

\section{APPENDIX: MATERIALS, METHODS, AND ADDITIONAL SUPPORTING INFORMATION}

This Appendix provides additional information on the Raman vertex calculation and the data analysis as well as further data taken with different wavelengths and $\mathrm{URu}_{2} \mathrm{Si}_{2}$ samples.

\section{Raman vertex calculation}

The Raman scattering rate depends on an effective (polarization-dependent) charge density matrix $\rho^{\lambda}$. Neglecting resonant processes and decomposing the light-scattering tensor elements into the basis function of the irreducible point group of the lattice $D_{4 h}, \rho^{\lambda}$ can be written as $[59,60]$

$$
\rho^{\lambda}=\sum_{\mathbf{k}, \sigma, m, n} \gamma_{m, n}^{\lambda}(\mathbf{k}) c_{\mathbf{k}, m, \sigma}^{\dagger} c_{\mathbf{k}, n, \sigma}
$$

where $\gamma_{m, n}^{\lambda}$ is the Raman vertex between bands $m$ and $n$ in the $\lambda$ symmetry $\left(A_{1 g}, B_{2 g}, \ldots\right)$ and $c_{\mathbf{k}, m(n) \sigma}^{(\dagger)}$ is the annihilation (creation) operator of an electron with momentum $\mathbf{k}$ with spin $\sigma$ in band $m$. The $\gamma_{m, n}^{\lambda}$ is related to the basis of the orbital by

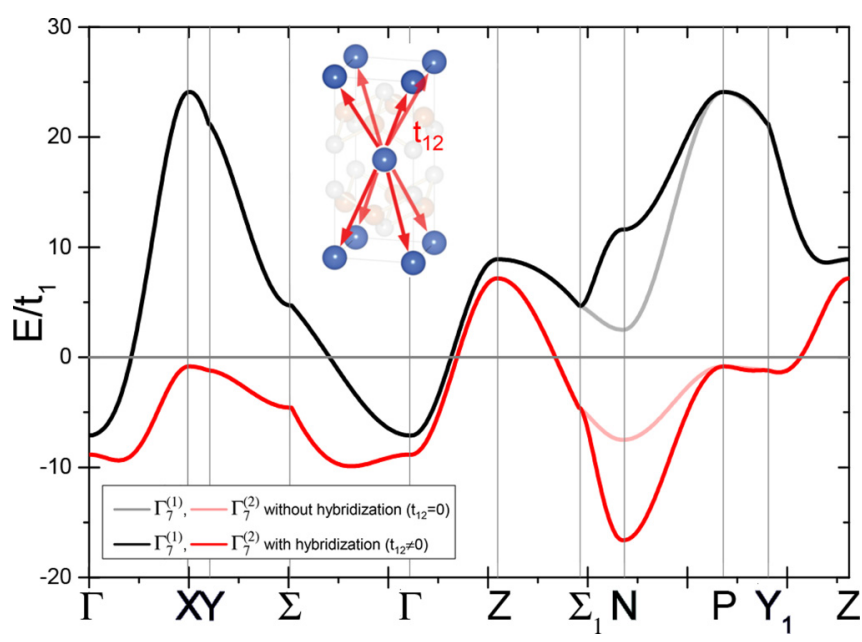

FIG. 4. Calculated electronic dispersion of $\Gamma_{7}^{(1)}$ and $\Gamma_{7}^{(2)}$ with hybridization and without hybridization (light colors). The parameters used here, in the hybridization case, are $t_{1}=t_{2}=1.56 \mathrm{meV}$, $t_{1}^{\prime}=4.52 \mathrm{meV}, t_{2}^{\prime}=0 \mathrm{meV}, t_{1}^{\prime \prime}=1.95 \mathrm{meV}, t_{2}^{\prime \prime}=1.3 \mathrm{meV}, \mu=2.6$ $\mathrm{meV}, \Delta=18.21 \mathrm{meV}$, and $t_{12}=3.64 \mathrm{meV}$.

the relation

$$
\gamma_{m, n}^{\lambda}=\sum_{\mu, \nu} \gamma_{\mu, \nu}^{\lambda}(\mathbf{k}) a_{\mu, n}^{\star} a_{v, m}
$$

with $\gamma_{\mu, \nu}^{\lambda}$ being the Raman vertex defined in the $\mu$-, $v$-orbital basis and $a_{\mu, n}$ being the rotation matrix between the orbital and the band basis. We consider intraband and also interband transitions, taking into account the orbital content of each band, as described in Ref. [60].

The Raman vertex $\gamma_{\mu, v}^{\lambda}$ in the effective-mass approximation [59] is given as

$$
\gamma_{\mu, v}^{\lambda}(\mathbf{k}, q \rightarrow 0)=\frac{m}{\hbar^{2}} \sum_{i, j} \mathbf{e}_{S}^{i *}\left(\frac{\partial^{2} \epsilon_{\mu, v}(\mathbf{k})}{\partial k_{i} \partial k_{j}}\right) \mathbf{e}_{L}^{j} .
$$

Here, the vertex value depends on the curvature of the energy band dispersion $\epsilon_{\mu, v}(\mathbf{k})$ together with the light polarization vectors $\mathbf{e}_{L}$ and $\mathbf{e}_{S}$ (the laser and scattered light polarizations, respectively). The combinations of directions $i$ and $j$ which contribute to the Raman scattering for each symmetry $\lambda$ are given in Ref. [59].

From relations (A2) and (A3), we calculated numerically the intraband and interband Raman vertices $\gamma_{m, n}^{\lambda}$ using the tight-binding electronic band structure developed on the $\Gamma_{7}^{(1)}$ and $\Gamma_{7}^{(2)}$ electronic $f$ states of uranium [61,62]. The band dispersions used and Raman vertices obtained are represented, respectively, in Figs. 4 and 5.

The Kondo lattice effect has been introduced as a mixing between the two $\Gamma_{7}$ doublets whose contribution to the Hamiltonian depends on a hopping term $t_{12}$ between the central and corner uranium sites. Geometrically, this $t_{12}$ hopping is expected to involve hybridization with the $\mathrm{Ru} d$ orbitals. This hybridization has been adjusted to fit the $a b$ initio Fermi surface $[62,63]$. The hybridization modifies the band dispersion around the $N$ point (Fig. 4). 


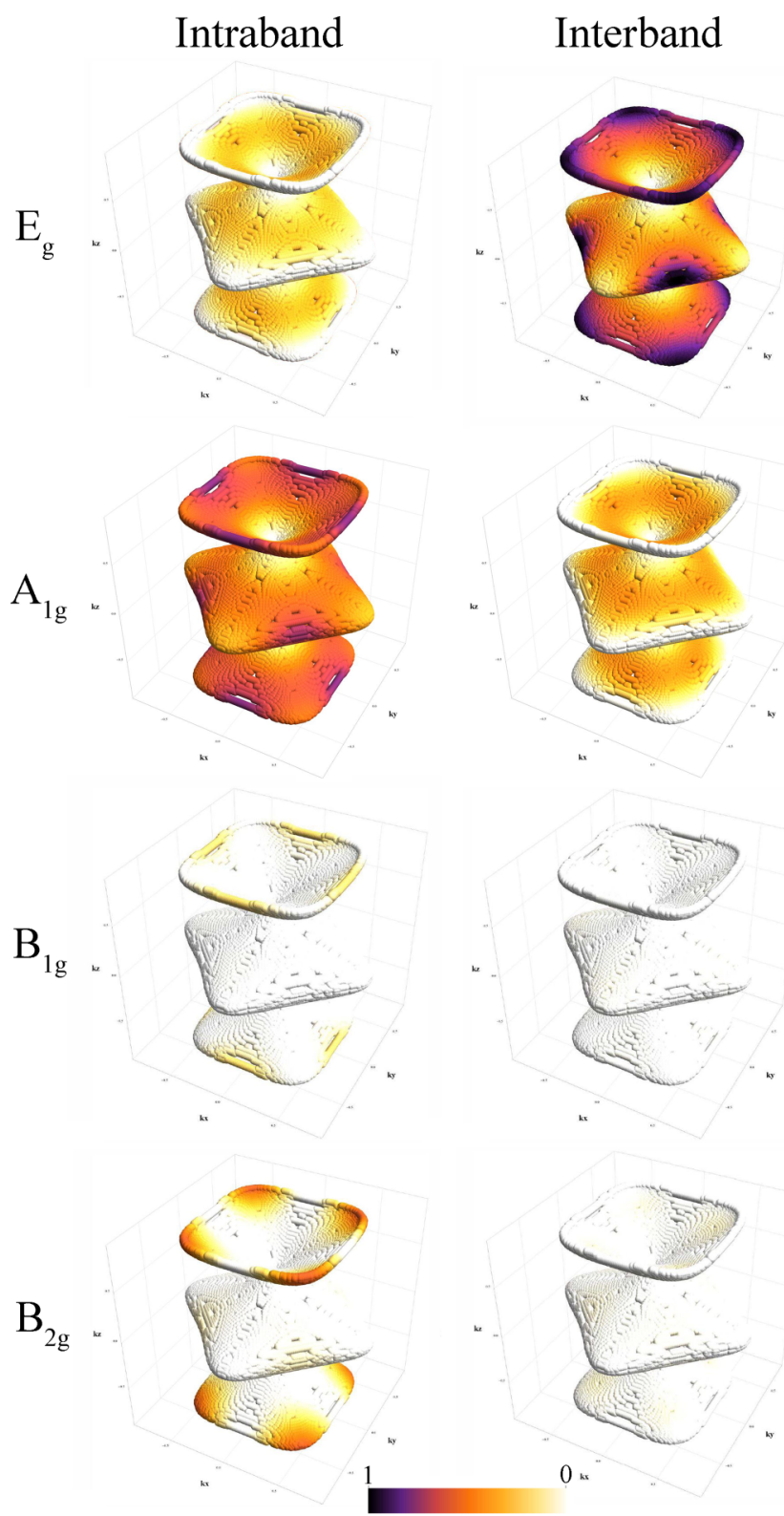

FIG. 5. Raman vertex magnitude at the Fermi surface for intraand interband transitions calculated in the effective-mass approximation. The amplitude of each vertex is normalized by the maximal amplitude of the $E_{g}$ interband vertex. The vertex value is maximum and minimum, respectively, for dark and white zones of the Fermi surface.

\section{Wavelength dependence}

All our results have been checked with both 532- and 647-nm laser lines. Figure 6 shows Raman spectra of $\mathrm{URu}_{2} \mathrm{Si}_{2}$ in the $\left[E_{g}\right]$ and $\left[A_{1 g}+B_{2 g}\right]$ symmetries at $647 \mathrm{~nm}$ and the $\left[A_{2 g}+B_{1 g}\right]$ symmetries at $532 \mathrm{~nm}$. In the $E_{g}$ symmetry, we observe a depletion in the electronic continuum below $700 \mathrm{~cm}^{-1}$ when the temperature decreases below $\sim 100 \mathrm{~K}$. This depletion is a clear signature of the Kondo pseudogap opening as observed at $532 \mathrm{~nm}$. Although our spectra are noisier in the $A_{1 g}$ symmetry, we still observe a slight depletion in the electronic continuum from room temperature. These two results at $647 \mathrm{~nm}$ are consistent with measurements at
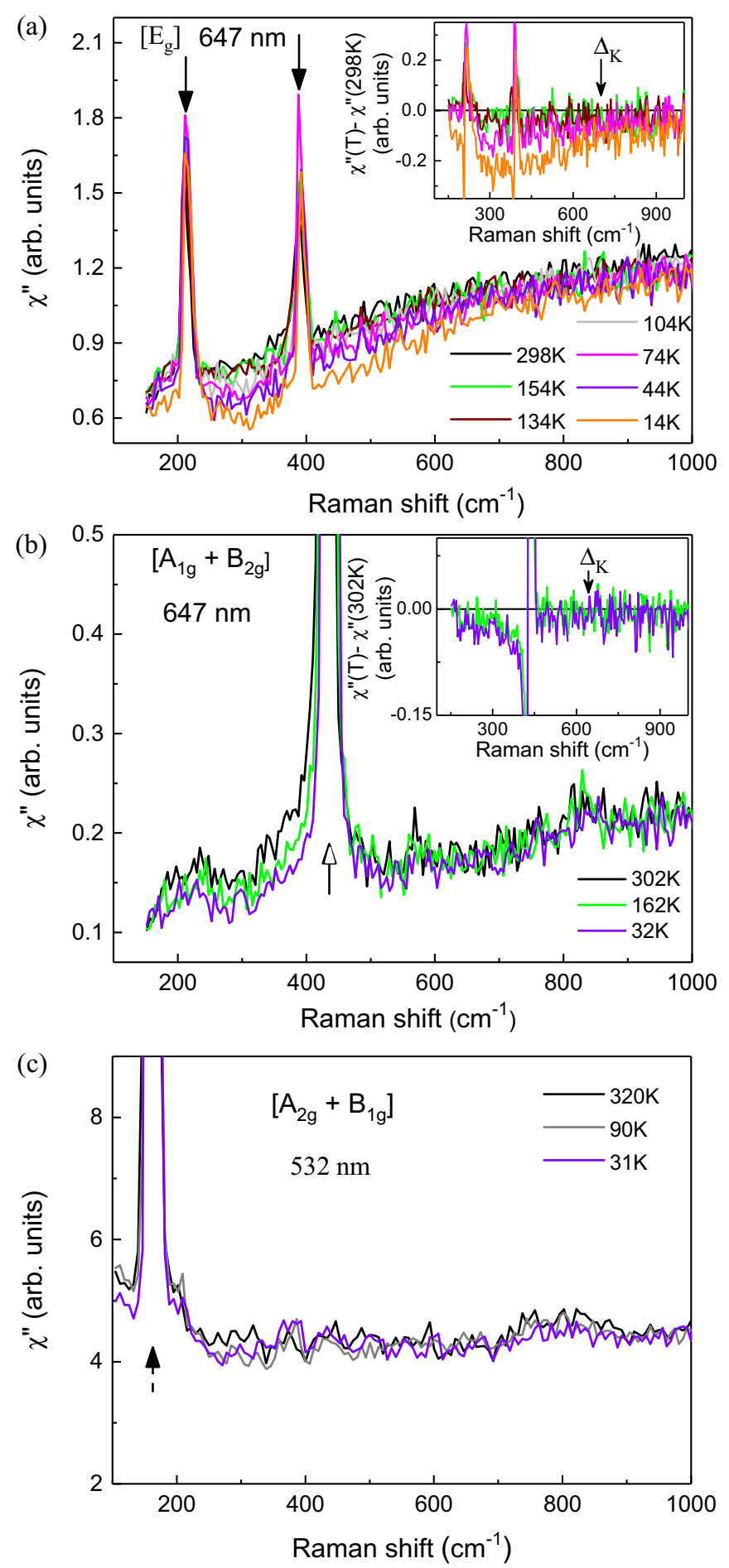

FIG. 6. Raman spectra of $\mathrm{URu}_{2} \mathrm{Si}_{2}$ in the (a) $E_{g}$ and (b) $A_{1 g}+B_{2 g}$ symmetries obtained with a laser line at $647 \mathrm{~nm}$ and (c) $A_{2 g}+B_{1 g}$ symmetries obtained with a laser line at $532 \mathrm{~nm}$. Insets in (a) and (b) show the subtracted Raman responses $\chi^{\prime \prime}(T)-\chi^{\prime \prime}\left(T_{\text {ambient }}\right)$. The laser heating is included in the reported temperatures. Solid arrows with a solid line, open arrows with a solid line, and solid arrows with a dashed line indicate, respectively, the $E_{g}, A_{1 g}$, and $B_{1 g}$ phonon modes.

$532 \mathrm{~nm}$ in the main text. In addition, since the penetration of light into the sample at $647 \mathrm{~nm}$ is deeper than at $532 \mathrm{~nm}$, we 


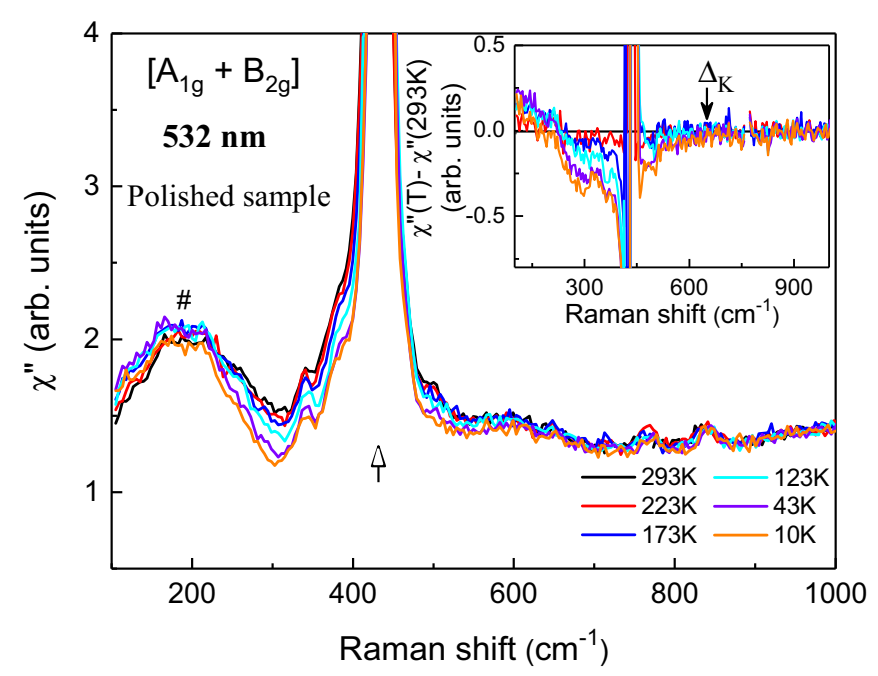

FIG. 7. Raman spectra of $\mathrm{URu}_{2} \mathrm{Si}_{2}$ in the $A_{1 g}+B_{2 g}$ symmetry for a polished sample. Insets show the subtracted Raman responses $\chi^{\prime \prime}(T)-\chi^{\prime \prime}\left(T_{293 K}\right)$. The laser heating is included in the reported temperatures. The open arrow indicates the $A_{1 g}$ phonon mode. The symbol \# denotes an extrinsic bump depending on the surface quality (see text).

confirm that the depletions seen in the electronic continuum in the $E_{g}$ and $A_{1 g}$ symmetries are an intrinsic bulk effect and do not arise from a surface artifact. Finally, no pseudogap opening is observed in the $\left[A_{2 g}+B_{1 g}\right]$ symmetries at $532 \mathrm{~nm}$ [Fig. 6(c)], in agreement with the result presented in the main text at $647 \mathrm{~nm}$.

\section{Cleaved versus polished samples}

Figure 7 presents Raman spectra of $\mathrm{URu}_{2} \mathrm{Si}_{2}$ in the $\left[A_{1 g}+\right.$ $B_{2 g}$ ] symmetry for a polished sample at several temperatures. A clear depletion in the electronic continuum occurs below $\sim 600 \mathrm{~cm}^{-1}$ with decreasing temperature (see inset in Fig. 7). This result is in total agreement with results shown for cleaved samples in the main text. Only the large bump centered at $200 \mathrm{~cm}^{-1}$ seems to be more intense in the polished samples. The energy of this bump is independent of temperature, and its intensity is lower for measurements performed at $647 \mathrm{~nm}$ than at $532 \mathrm{~nm}$. We thus conclude that this broad peak is linked to the surface quality and is not intrinsic. Here, we confirm that the result obtained in $A_{1 g}$ symmetry with a freshly cleaved sample is reproducible with polished samples along the $(a b)$ plane. Therefore, together with the consistent results obtained using different laser lines (see Sec. A2), we are convinced that the Kondo pseudogap opening observed in $E_{g}$ symmetry, which can be probed only in polished samples in the $(a c)$ plane, is intrinsic.

\section{Extraction of the spectral weight}

Since the Raman responses in the $\left[B_{1 g}+B_{2 g}\right],\left[A_{1 g}+B_{2 g}\right]$, and $\left[E_{g}\right]$ symmetries probed at $532 \mathrm{~nm}$ have been performed exactly in the same experimental conditions (bar the rotation of a polarizer), their intensities can be compared directly. The $\left[A_{2 g}+B_{1 g}\right]$ response, measured with a $647-\mathrm{nm}$ excitation laser line, has been normalized with respect to the electronic background of the $\left[A_{2 g}+B_{1 g}\right]$ symmetry response probed with the 532-nm laser line. The phonon modes were removed from the electronic background. The error bars on the spectral weight were evaluated by integrating the noise of the subtracted spectra over the same energy range.
[1] J. Kondo, Prog. Theor. Phys. 32, 37 (1964).

[2] A. C. Hewson, The Kondo Problem to Heavy Fermions (Cambridge University Press, Cambridge, UK, 1997).

[3] Y.-f. Yang and D. Pines, Proc. Natl. Acad. Sci. USA 109, E3060 (2012).

[4] G. Lonzarich, D. Pines, and Y.-f. Yang, Rep. Prog. Phys. 80, 024501 (2017).

[5] H. Ikeda and K. Miyake, J. Phys. Soc. Jpn. 65, 1769 (1996).

[6] H. Weber and M. Vojta, Phys. Rev. B 77, 125118 (2008).

[7] P. Ghaemi and T. Senthil, Phys. Rev. B 75, 144412 (2007).

[8] S. Burdin and V. Zlatić, Phys. Rev. B 79, 115139 (2009).

[9] P. S. Riseborough and J. M. Lawrence, Rep. Prog. Phys. 79, 084501 (2016).

[10] P. Nozières, J. Phys. Soc. Jpn. 74, 4 (2005).

[11] C. Lacroix and M. Cyrot, Phys. Rev. B 20, 1969 (1979).

[12] K. S. Burch, S. V. Dordevic, F. P. Mena, A. B. Kuzmenko, D. van der Marel, J. L. Sarrao, J. R. Jeffries, E. D. Bauer, M. B. Maple, and D. N. Basov, Phys. Rev. B 75, 054523 (2007).

[13] J. A. Mydosh and P. M. Oppeneer, Rev. Mod. Phys. 83, 1301 (2011).

[14] J. A. Mydosh, Philos. Mag. 94, 3640 (2014).

[15] D. A. Bonn, J. D. Garrett, and T. Timusk, Phys. Rev. Lett. 61, 1305 (1988).
[16] U. Nagel, T. Uleksin, T. Room, R. P. S. M. Lobo, P. Lejay, C. C. Homes, J. S. Hall, A. W. Kinross, S. K. Purdy, T. Munsie, T. J. Williams, G. M. Luke, and T. Timusk, Proc. Natl. Acad. Sci. USA 109, 19161 (2012).

[17] W. T. Guo, Z. G. Chen, T. J. Williams, J. D. Garrett, G. M. Luke, and N. L. Wang, Phys. Rev. B 85, 195105 (2012).

[18] J. Levallois, F. Lévy-Bertrand, M. K. Tran, D. Stricker, J. A. Mydosh, Y.-K. Huang, and D. van der Marel, Phys. Rev. B 84, 184420 (2011).

[19] W. K. Park, P. H. Tobash, F. Ronning, E. D. Bauer, J. L. Sarrao, J. D. Thompson, and L. H. Greene, Phys. Rev. Lett. 108, 246403 (2012).

[20] N. Bachar, D. Stricker, S. Muleady, K. Wang, J. A. Mydosh, Y. K. Huang, and D. van der Marel, Phys. Rev. B 94, 235101 (2016).

[21] A. R. Schmidt, M. H. Hamidian, P. Wahl, F. Meier, A. V. Balatsky, J. D. Garrett, T. J. Williams, G. M. Luke, and J. C. Davis, Nature (London) 465, 570 (2010).

[22] P. Aynajian, E. H. da Silva Neto, C. V. Parker, Y. Huang, A. Pasupathy, J. Mydosh, and A. Yazdani, Proc. Natl. Acad. Sci. USA 107, 10383 (2010).

[23] F. L. Boariu, C. Bareille, H. Schwab, A. Nuber, P. Lejay, T. Durakiewicz, F. Reinert, and A. F. Santander-Syro, Phys. Rev. Lett. 110, 156404 (2013). 
[24] G. W. Scheerer, W. Knafo, D. Aoki, G. Ballon, A. Mari, D. Vignolles, and J. Flouquet, Phys. Rev. B 85, 094402 (2012).

[25] A. E. Sikkema, W. J. L. Buyers, I. Affleck, and J. Gan, Phys. Rev. B 54, 9322 (1996).

[26] V. E. Mineev and M. E. Zhitomirsky, Phys. Rev. B 72, 014432 (2005).

[27] K. Haule and G. Kotliar, Nat. Phys. 5, 796 (2009).

[28] C. Pépin, M. R. Norman, S. Burdin, and A. Ferraz, Phys. Rev. Lett. 106, 106601 (2011).

[29] P. S. Riseborough, B. Coqblin, and S. G. Magalhães, Phys. Rev. B 85, 165116 (2012).

[30] Y. Dubi and A. V. Balatsky, Phys. Rev. Lett. 106, 086401 (2011).

[31] P. Chandra, P. Coleman, and R. Flint, Nature (London) 493, 621 (2013).

[32] Y. Kasahara, T. Iwasawa, H. Shishido, T. Shibauchi, K. Behnia, Y. Haga, T. D. Matsuda, Y. Onuki, M. Sigrist, and Y. Matsuda, Phys. Rev. Lett. 99, 116402 (2007).

[33] I. Kawasaki, I. Watanabe, A. Hillier, and D. Aoki, J. Phys. Soc. Jpn. 83, 094720 (2014).

[34] A. Akbari and P. Thalmeier, Phys. Rev. B 90, 224511 (2014).

[35] D. Aoki, F. Bourdarot, E. Hassinger, G. Knebel, A. Miyake, S. Raymond, V. Taufour, and J. Flouquet, J. Phys.: Condens. Matter 22, 164205 (2010).

[36] W. Hayes and R. Loudon, Scattering of Light by Crystals, Dover Books on Physics (Dover, Mineola, NY, 2004).

[37] R. Hackl, R. Kaiser, and S. Schicktanz, J. Phys. C 16, 1729 (1983).

[38] A. A. Maksimov, A. V. Puchkov, I. I. Tartakovskii, V. B. Timofeev, D. Reznik, and M. V. Klein, Solid State Commun. 81, 407 (1992).

[39] A. Mialitsin, Ph.D. thesis, Rutgers, the State University of New Jersey, 2010.

[40] F. G. Aliev, V. Kovachik, V. V. Moshchalkov, V. V. Pryadun, N. E. Alekseevskii, A. V. Mitin, N. Agrait, S. Vieira, and R. Villar, J. Low Temp. Phys. 85, 359 (1991).

[41] J. Buhot, M. A. Méasson, Y. Gallais, M. Cazayous, A. Sacuto, F. Bourdarot, S. Raymond, G. Lapertot, D. Aoki, L. P. Regnault, A. Ivanov, P. Piekarz, K. Parlinski, D. Legut, C. C. Homes, P. Lejay, and R. P. S. M. Lobo, Phys. Rev. B 91, 035129 (2015).

[42] J. Buhot, Y. Gallais, M. Cazayous, A. Sacuto, P. Piekarz, G. Lapertot, D. Aoki, and M.-A. Méasson, Phys. B (Amsterdam, Neth.) 506, 19 (2017).

[43] T. T. M. Palstra, A. A. Menovsky, J. van den Berg, A. J. Dirkmaat, P. H. Kes, G. J. Nieuwenhuys, and J. A. Mydosh, Phys. Rev. Lett. 55, 2727 (1985).

[44] T. T. M. Palstra, A. A. Menovsky, and J. A. Mydosh, Phys. Rev. B 33, 6527 (1986).

[45] S. L. Cooper, P. Nyhus, S. Yoon, Z. Fisk, and J. Sarrao, in Proceedings of the International Conference on Low Energy Electrodynamics in Solids, special issue of Phys. B (Amsterdam, Neth.) 244, 133 (1998).
[46] S. V. Dordevic, D. N. Basov, N. R. Dilley, E. D. Bauer, and M. B. Maple, Phys. Rev. Lett. 86, 684 (2001).

[47] L. Degiorgi, Rev. Mod. Phys. 71, 687 (1999).

[48] Z. Fisk, J. L. Sarrao, S. L. Cooper, P. Nyhus, G. S. Boebinger, A. Passner, and P. C. Canfield, in Proceedings of the International Conference on Strongly Correlated Electron Systems, special issue of Phys. B (Amsterdam, Neth.)223-224, 409 (1996).

[49] R. P. S. M. Lobo, J. Buhot, M. A. Méasson, D. Aoki, G. Lapertot, P. Lejay, and C. C. Homes, Phys. Rev. B 92, 045129 (2015).

[50] J. Schoenes, C. Schonenberger, J. J. M. Franse, and A. A. Menovsky, Phys. Rev. B 35, 5375 (1987).

[51] I. Giannakis, J. Leshen, M. Kavai, S. Ran, C.-J. Kang, S. R. Saha, Y. Zhao, Z. Xu, J. W. Lynn, L. Miao, L. A. Wray, G. Kotliar, N. P. Butch, and P. Aynajian, Sci. Adv. 5, eaaw9061 (2019).

[52] Q. Y. Chen, X. B. Luo, D. H. Xie, M. L. Li, X. Y. Ji, R. Zhou, Y. B. Huang, W. Zhang, W. Feng, Y. Zhang, L. Huang, Q. Q. Hao, Q. Liu, X. G. Zhu, Y. Liu, P. Zhang, X. C. Lai, Q. Si, and S. Y. Tan, Phys. Rev. Lett. 123, 106402 (2019).

[53] S. Burdin, A. Georges, and D. R. Grempel, Phys. Rev. Lett. 85, 1048 (2000).

[54] L. Degiorgi, F. B. B. Anders, and G. Grüner, Eur. Phys. J. B 19, 167 (2001).

[55] J. K. Freericks and T. P. Devereaux, Phys. Rev. B 64, 125110 (2001).

[56] J. K. Freericks, T. P. Devereaux, M. Moraghebi, and S. L. Cooper, Phys. Rev. Lett. 94, 216401 (2005).

[57] J. K. Freericks, T. P. Devereaux, R. Bulla, and T. Pruschke, Phys. Rev. B 67, 155102 (2003).

[58] J. Buhot, M.-A. Méasson, Y. Gallais, M. Cazayous, A. Sacuto, G. Lapertot, and D. Aoki, Phys. Rev. Lett. 113, 266405 (2014).

[59] T. P. Devereaux and R. Hackl, Rev. Mod. Phys. 79, 175 (2007).

[60] B. Valenzuela, M. J. Calderón, G. León, and E. Bascones, Phys. Rev. B 87, 075136 (2013).

[61] P. Thalmeier and T. Takimoto, Phys. Rev. B 83, 165110 (2011).

[62] J. G. Rau and H.-Y. Kee, Phys. Rev. B 85, 245112 (2012).

[63] P. M. Oppeneer, J. Rusz, S. Elgazzar, M.-T. Suzuki, T. Durakiewicz, and J. A. Mydosh, Phys. Rev. B 82, 205103 (2010).

[64] P. Thalmeier, T. Takimoto, and H. Ikeda, Philos. Mag. 94, 3863 (2014)..

[65] H.-H. Kung, R. E. Baumbach, E. D. Bauer, V. K. Thorsmølle, W.-L. Zhang, K. Haule, J. A. Mydosh, and G. Blumberg, Science 347, 1339 (2015).

[66] S. L. Cooper, M. V. Klein, M. B. Maple, and M. S. Torikachvili, Phys. Rev. B 36, 5743 (1987).

[67] R. Flint, P. Chandra, and P. Coleman, J. Phys. Soc. Jpn. 83, 061003 (2014). 